JOURNAL OF THE

AMERICAN MATHEMATICAL SOCIETY

Volume 12, Number 3, Pages 839-848

S 0894-0347(99)00301-X

Article electronically published on April 13, 1999

\title{
PYTHAGORAS NUMBERS OF FIELDS
}

\author{
DETLEV W. HOFFMANN
}

\section{INTRODUCTION}

The study of sums of squares in a ring or a field is a classic topic in algebra and number theory. In this context, several questions arise naturally. For example, which elements can be represented as sums of squares, and if an element can be written as a sum of squares, how many squares are actually needed?

For instance, for an integer $n$ to be a sum of squares of integers, we must obviously have that $n \geq 0$, and it is well known that each such $n$ can in fact be written as a sum of four integer squares, the first published proof of this result being due to Lagrange. Furthermore, in general one needs four squares as, for example, 7 cannot be written as a sum of three integer squares. This result readily carries over to the rational numbers $\mathbb{Q}: x \in \mathbb{Q}$ is a sum of squares of elements in $\mathbb{Q}$ if and only if $x \geq 0$, and in this case $x$ can be written as a sum of $\leq 4$ squares. However, 7 cannot be written as a sum of three squares in $\mathbb{Q}$. If we define the Pythagoras number $p(R)$ of a ring $R$ to be the least integer $n$ (if such an integer exists) such that each sum of squares in $R$ can be written as a sum of $\leq n$ squares, then the above shows that $p(\mathbb{Z})=p(\mathbb{Q})=4$. For number fields $F$ in general, it follows readily from the Hasse-Minkowski theorem that $p(F) \leq 4$. If $R$ is an order in $F$, then $p(R)$ is finite but can grow arbitrarily large in the case of a totally real $F$ (cf. $[\mathrm{Pe}],[\mathrm{Sc}]$ ).

Hilbert's 17th problem is another classic example concerning sums of squares, the problem itself being essentially the following: Let $F=\mathbb{R}\left(X_{1}, \cdots, X_{n}\right)$ be the rational function field in $n$ variables over the real numbers $\mathbb{R}$, and let $f \in F$ be a rational function such that $f(a) \geq 0$ for all $a=\left(a_{1}, \cdots, a_{n}\right) \in \mathbb{R}^{n}$ where $f$ is defined ( $f$ is then called positive semi-definite). Is $f$ a sum of squares in $F$ ? (It can readily be shown that in order to be a sum of squares, $f$ must necessarily be positive semi-definite.) This problem has been given a positive answer by Artin in $1927[\mathrm{~A}]$. One can furthermore show that in this situation $p(F) \leq 2^{n}$, so that in particular $p\left(\mathbb{R}\left(X_{1}\right)\right)=2$ (note that for example $1+X_{1}^{2}$ is a sum of two squares which is not a square), and for $n=2$, it was shown by Cassels-Ellison-Pfister in $1971[\mathrm{CEP}]$ that $p\left(\mathbb{R}\left(X_{1}, X_{2}\right)\right)=4$. For $n \geq 3$ the precise value is not known.

For this and more on generalized versions of Hilbert's 17th problem, we refer to the exposition given in Chapters 6 and 7 of Pfister's beautiful book [Pf], where one also finds an account of the current state of knowledge regarding problems related to the Pythagoras number (also for rings). One question asked there was which

Received by the editors July 31, 1998 and, in revised form, February 12, 1999.

1991 Mathematics Subject Classification. Primary 11E04, 11E10, 11E25, $12 \mathrm{D} 15$.

Key words and phrases. Quadratic forms, sums of squares, formally real fields, Pythagoras number, $u$-invariant, Hasse number. 
positive integers can occur as Pythagoras numbers of fields (cf. [Pf, Ch. 7, Open problem 1.6]). It is this problem which we will attack in this paper.

Let $F$ be a field of characteristic $\neq 2$ and denote by $S(F)$ the set of nonzero sums of squares in $F$, i.e.

$$
S(F)=\left\{x \in F^{*} \mid \exists r \in \mathbb{N}, x_{1}, \cdots, x_{r} \in F^{*} \text { such that } x=\sum_{i=1}^{r} x_{i}^{2}\right\} .
$$

For $x \in S(F)$, we define its length $\ell(x)$ to be

$$
\ell(x)=\min \left\{r \in \mathbb{N} \mid \exists x_{1}, \cdots, x_{r} \in F^{*} \text { such that } x=\sum_{i=1}^{r} x_{i}^{2}\right\} .
$$

The Pythagoras number $p(F)$ of $F$ is then given by

$$
p(F)=\sup \{\ell(x) \mid x \in S(F)\} .
$$

Suppose first that $F$ is not formally real, i.e. -1 is a sum of squares in $F$. In this case, the level $s$ of $F$ is defined to be $s(F)=\ell(-1)$, and it is a well-known theorem due to Pfister that $s(F)$ will always be a 2-power, and that all 2-powers can be realized as levels of suitable fields (see [Pf, Ch. 3, Theorems 1.3, 1.4]). Using this, it is now easy to prove that for a nonformally real field $F$ with $s(F)=2^{m}$, one has $p(F)=2^{m}$ or $p(F)=2^{m}+1$ (cf. [Pf, Ch.7, Lemma 1.3]), and one can show that to each $m \in \mathbb{N} \cup\{0\}$ there exist nonformally real fields $F, F^{\prime}$ with $p(F)=2^{m}$, resp. $p\left(F^{\prime}\right)=2^{m}+1$ (cf. [Pf, Ch. 7, Prop. 1.5]).

It therefore remains to consider the case of a formally real field. Prestel [Pr] (cf. also [Pf, Ch. 7, Prop. 1.5']) has shown that to each $m \in \mathbb{N} \cup\{0\}$ there exist formally real fields $F, F^{\prime}, F^{\prime \prime}$ with $p(F)=2^{m}, p\left(F^{\prime}\right)=2^{m}+1, p\left(F^{\prime \prime}\right)=\infty$, resp. In fact, he shows that there exist uniquely ordered subfields $F, F^{\prime}, F^{\prime \prime}$ of the reals which will take these prescribed values, and $F$ and $F^{\prime}$ will in addition have finite transcendence degree over the rationals. It remained an open problem whether for formally real fields the Pythagoras number, if finite, would always be of the type $2^{m}$ or $2^{m}+1$.

We will show in the next section that this is not the case. In fact, we will prove

Theorem 1. Let $n \geq 1$ be an integer. Let $F_{0}$ be a formally real field. Then there exists a formally real field $F$ over $F_{0}$ with $p(F)=n$.

In the last section, we will give refinements of this construction to obtain to any given integers $n, m \geq 2$ and $k \geq 1$ with $2 m \geq 2^{k} \geq n$ a uniquely ordered field $F$ with $p(F)=n$ and $u(F)=\tilde{u}(F)=2 m$ (Theorem 2), and we will also give examples of uniquely ordered fields $F$ with $p(F)=n$ and $u(F)=\tilde{u}(F)=\infty$ (Theorem 3). Here, $u(F)$ (resp. $\tilde{u}(F)$ ) denotes the $u$-invariant (resp. the Hasse number) of $F$ defined as the supremum of the dimensions of all anisotropic forms over $F$ which are torsion in the Witt ring of $F$ (resp. which are indefinite with respect to each ordering on $F$ ). These refinements will be achieved by combining our method presented in the next section with Merkurjev's method of constructing fields which have as $u$-invariant any given even positive integer [M].

For the proof of Theorem 1, we will need certain facts from the algebraic theory of quadratic forms which can all be found in the standard references [L1] and [S]. For quadratic forms $\varphi$ and $\psi$ over $F, \varphi \perp \psi, \varphi \cong \psi, \varphi \sim \psi$ will denote orthogonal sum, isometry, and equivalence in the Witt ring $W F$, respectively. If $\varphi$ is a subform of $\psi$, i.e. $\psi \cong \varphi \perp \tau$ for a suitable form $\tau$, then we will write $\varphi \subset \psi$ for short.

A form of type $\left\langle 1,-a_{1}\right\rangle \otimes \cdots \otimes\left\langle 1,-a_{n}\right\rangle, a_{i} \in F^{*}$, will be called an $n$-fold Pfister form and we will use the short notation $\left\langle\left\langle a_{1}, \cdots, a_{n}\right\rangle\right\rangle$ to denote that form. The set of forms isometric (resp. similar) to $n$-fold Pfister forms will be denoted by 
$P_{n} F$ (resp. $G P_{n} F$ ). If $\pi$ is a Pfister form, we can write $\pi \cong\langle 1\rangle \perp \pi^{\prime}$ where $\pi^{\prime}$ is uniquely determined up to isometry. $\pi^{\prime}$ is called the pure part of $\pi$. A form $\varphi$ is called a Pfister neighbor if there exist $a \in F^{*}$ and $\pi \in P_{n} F$ for some $n$ such that $\varphi \subset a \pi$ and $2 \operatorname{dim} \varphi>\operatorname{dim} \pi=2^{n}$. In this case, we say that $\varphi$ is a Pfister neighbor of $\pi$. Pfister forms have several very nice properties. They are multiplicative in the sense that if $\pi$ is a Pfister form, then $a \in F^{*}$ is represented by $\pi$ if and only if $\pi \cong a \pi$. They are either anisotropic or hyperbolic. If $\varphi$ is a Pfister neighbor of a Pfister form $\pi$ and a Pfister form $\tau$, then in fact $\pi \cong \tau$ (this can be shown using the Cassels-Pfister subform theorem [S, Ch. 4, Theorem 5.4], see below, or the ArasonPfister Hauptsatz [S, Ch. 4, Theorem 5.6]). We have that this Pfister neighbor $\varphi$ is anisotropic iff $\pi$ is anisotropic, and if $\varphi$ represents $a \in F^{*}$, then the multiplicativity of $\pi$ implies $\varphi \subset a \pi$. If $\psi$ is the uniquely determined form of dimension $<\operatorname{dim} \varphi$ such that $a \pi \cong \varphi \perp \psi$, then $\psi$ is called the complementary form of the Pfister neighbor $\varphi$ (the uniqueness of $\psi$ is a consequence of Witt cancellation) and $\operatorname{dim} \psi$ is called the codimension of $\varphi$.

Now let $\varphi$ be a form of dimension $\geq 3$ over $F$. The function field $F(\varphi)$ of $\varphi$ is the function field of the projective quadric defined by the equation $\varphi=0 . F(\varphi) / F$ is of transcendence degree $n-2$ over $F$, and it is purely transcendental if and only if $\varphi$ is isotropic over $F$. If $\varphi \cong\langle a,-a d\rangle$ with $a, d \in F^{*}$, then we put $F(\varphi)=F(\sqrt{d})$ if $d \notin F^{* 2}$, and $F(\varphi)=F$ if $d \in F^{* 2}$ (i.e. $\varphi$ is isometric to the hyperbolic plane $\mathbb{H}=\langle 1,-1\rangle)$. We also put $F(\varphi)=F$ if $\operatorname{dim} \varphi=1$. The Cassels-Pfister subform theorem states that if an anisotropic form $\psi$ over $F$ becomes hyperbolic over the function field $F(\varphi)$ of another form $\varphi$ over $F$, then for any $a \in F^{*}$ represented by $\varphi$ and any $b \in F^{*}$ represented by $\psi$ we have $a b \varphi \subset \psi$.

\section{Proof of Theorem 1}

Our proof is based on a result in which the notion of excellent forms will play an important role. This notion has been introduced by Knebusch $[\mathrm{K}]$. By definition, forms of dimension $\leq 1$ are excellent of height 0 . A form $\varphi$ is called excellent of height $h \geq 1$ if $\varphi$ is a Pfister neighbor and its complementary form is an excellent form of height $h-1$. So if $\varphi=\varphi_{0}$ is excellent of height $h \geq 1$, then $\varphi_{0}$ is a Pfister neighbor with complementary form $\varphi_{1}$, i.e. $\varphi_{0} \perp \varphi_{1} \in G P_{m_{1}} F$ for some $m_{1}, \varphi_{1}$ is a Pfister neighbor with complementary form $\varphi_{2}$, i.e. $\varphi_{1} \perp \varphi_{2} \in G P_{m_{2}} F$ for some $m_{2}<m_{1}$, and so on. The $\varphi_{i}, 1 \leq i \leq h$, are called the higher complementary forms of $\varphi_{0}$. Note that if $\varphi_{0}$ is anisotropic, then the associated Pfister form $\varphi_{0} \perp \varphi_{1}$ is anisotropic, hence $\varphi_{1}$ is anisotropic and by induction all $\varphi_{i}$ and all the Pfister forms $\varphi_{i} \perp \varphi_{i+1}$ are anisotropic. A typical example of an excellent form is the quadratic form given by a sum of squares, $\varphi_{0} \cong\langle 1, \cdots, 1\rangle$. It will be a Pfister neighbor of a Pfister form of type $\langle\langle-1, \cdots,-1\rangle\rangle$, and one readily checks that all higher complementary forms will again be sums of squares.

Lemma 1. Let $\varphi_{0}$ be an anisotropic excellent form of height $h \geq 2$ with higher complementary forms $\varphi_{i}, 1 \leq i \leq h$. Then $\varphi_{0} \supset \varphi_{2} \supset \varphi_{4} \supset \cdots$ and $\varphi_{1} \supset \varphi_{3} \supset$ $\varphi_{5} \supset \cdots$.

Proof. Let $\pi_{1} \in G P_{m_{1}} F$ and $\pi_{2} \in G P_{m_{2}} F, 1 \leq m_{2}<m_{1}$, such that $\varphi_{0} \perp \varphi_{1} \cong \pi_{1}$ and $\varphi_{1} \perp \varphi_{2} \cong \pi_{2}$. (Note that $1 \leq m_{2}$ follows from $h \geq 2$.) In the Witt ring, we thus get

$$
\varphi_{0}-\varphi_{2} \sim \pi_{1}-\pi_{2} .
$$


The left hand side has dimension $\operatorname{dim} \varphi_{0}+\operatorname{dim} \varphi_{2}<\operatorname{dim} \varphi_{0}+\operatorname{dim} \varphi_{1}=2^{m_{1}}$, and the right hand side has dimension $2^{m_{1}}+2^{m_{2}}$. Hence, the Witt index of $\pi_{1} \perp-\pi_{2}$ must be $>2^{m_{2}-1}$. It will also be $\leq 2^{m_{2}}$ since both $\pi_{1}$ and $\pi_{2}$ are anisotropic. Since this Witt index must be a 2-power (cf. [EL, Prop. 4.4] or [H2, Lemma 3.2]), it equals $2^{m_{2}}$. This implies that $\varphi_{1} \perp \varphi_{2} \cong \pi_{2} \subset \pi_{1} \cong \varphi_{0} \perp \varphi_{1}$. Witt cancellation yields $\varphi_{2} \subset \varphi_{0}$. The full statement now follows easily from the fact that all the $\varphi_{i}$ are excellent.

Remark. In the situation of this lemma, one can show that there exist $x_{i} \in F^{*}$ such that $\varphi_{i+1} \subset x_{i} \varphi_{i}, 0 \leq i \leq h-1$. We leave this as an exercise for the reader. This implies in particular that there exists an $x \in F^{*}$ such that $x \varphi_{2} \subset \varphi_{0}$. However, for later purposes, we will need the fact that one can choose $x=1$.

The next lemma is essentially due to Izhboldin [I, Prop. 7.4]. We will give a proof for the reader's convenience.

Lemma 2. Let $\varphi_{0}$ be a Pfister neighbor of dimension $2^{m}+r$ with $1 \leq r \leq 2^{m}-1$, let $x \in F^{*}$ and let $\varphi=\varphi_{0} \perp\langle x\rangle$. Let $\varphi_{1}$ be the complementary form of $\varphi_{0}$ and $\pi \in G P_{m+1} F$ such that $\varphi_{0} \perp \varphi_{1} \cong \pi$. Let $\sigma=\varphi_{1} \perp\langle-x\rangle$. Then the following holds:

(i) Let $L / F$ be any field extension. Then $\varphi_{L}$ is a Pfister neighbor iff $\varphi_{L} \subset \pi_{L}$ iff $\sigma_{L}$ is isotropic.

(ii) If $\varphi$ is anisotropic, then $\varphi_{F(\sigma)}$ is an anisotropic Pfister neighbor. In particular, $\varphi_{F(\sigma)} \subset \pi_{F(\sigma)}$ and $\pi_{F(\sigma)}$ is anisotropic.

Proof. (i) If $\varphi_{L}$ is a Pfister neighbor, then it is a Pfister neighbor of some form in $P_{m+1} L$. This Pfister form must be similar to $\pi_{L} \in G P_{m+1} L$ because both Pfister neighbors $\varphi_{L}$ and $\pi_{L}$ have the Pfister neighbor $\left(\varphi_{0}\right)_{L}$ in common. Multiplicativity of Pfister forms then in fact implies $\varphi_{L} \subset \pi_{L}$. The converse is obvious.

By Witt cancellation, we have that $\varphi_{L} \cong\left(\varphi_{0} \perp\langle x\rangle\right)_{L} \subset \pi_{L} \cong\left(\varphi_{0} \perp \varphi_{1}\right)_{L}$ if and only if $\left(\varphi_{1}\right)_{L}$ represents $x$, i.e. if and only if $\left(\varphi_{1} \perp\langle-x\rangle\right)_{L}=\sigma_{L}$ is isotropic.

(ii) Suppose now that $\varphi$ is anisotropic. Since $\sigma_{F(\sigma)}$ is isotropic, it follows from (i) that $\varphi_{F(\sigma)} \subset \pi_{F(\sigma)}$, and it suffices to show that $\pi_{F(\sigma)}$ is anisotropic. Now $\pi$ is anisotropic as the Pfister neighbor $\varphi_{0}$ contained in $\pi$ is anisotropic. Suppose that $\pi_{F(\sigma)}$ is isotropic and hence hyperbolic. Then $\sigma$ is similar to a subform of $\pi$ by the Cassels-Pfister subform theorem. The fact that $\varphi_{1} \subset \pi$ and $\varphi_{1} \subset \sigma$ now implies that $\sigma \cong \varphi_{1} \perp\langle-x\rangle \subset \pi \cong \varphi_{0} \perp \varphi_{1}$. Witt cancellation yields that $\varphi_{0}$ represents $-x$, i.e. $\varphi=\varphi_{0} \perp\langle x\rangle$ is isotropic, a contradiction.

Proposition. Let $\varphi_{0}$ (resp. $\left.\psi_{0}\right)$ be excellent forms with $\operatorname{dim} \psi_{0}>\operatorname{dim} \varphi_{0}$. Let $x, y \in F^{*}$ and let $\varphi=\varphi_{0} \perp\langle x\rangle$ and $\psi=\psi_{0} \perp\langle y\rangle$. Let $m \geq 0$ such that $2^{m}+1 \leq \operatorname{dim} \psi \leq 2^{m+1}$. Suppose that $\varphi$ is anisotropic and that one of the following conditions holds:

(i) $\operatorname{dim} \varphi \leq 2^{m}$, or

(ii) $2^{m}+1 \leq \operatorname{dim} \varphi<\operatorname{dim} \psi \leq 2^{m+1}$ and $\psi$ is not a Pfister neighbor.

Then $\varphi_{F(\psi)}$ is anisotropic.

Proof. We will use induction on $m$. If we are in the situation of (i), the anisotropy of $\varphi_{F(\psi)}$ follows from [H1, Theorem 1]. Note that if $m=0$, then we are certainly in the situation of (i). 
So suppose that (ii) holds. If $\psi$ is isotropic, then $F(\psi) / F$ is purely transcendental and therefore $\varphi$ stays anisotropic over $F(\psi)$. Hence we may assume that $\psi$ is anisotropic.

Suppose first that $\varphi$ is a Pfister neighbor, say, of $\pi \in P_{m+1} F$. Note that the anisotropy of $\varphi$ implies that $\pi$ is anisotropic. If $\varphi_{F(\psi)}$ were isotropic, then $\pi$ would become hyperbolic over $F(\psi)$, which, by the Cassels-Pfister subform theorem, would imply that $\psi$ would be similar to a subform of $\pi$. Since $2 \operatorname{dim} \psi>\operatorname{dim} \pi, \psi$ would be a Pfister neighbor of $\pi$, a contradiction to our assumption. Therefore, if $\varphi$ is a Pfister neighbor, then $\varphi_{F(\psi)}$ is anisotropic.

Note that if $\operatorname{dim} \varphi=2^{m}+1$, then $\varphi$ is in fact a Pfister neighbor. This is because $\varphi=\varphi_{0} \perp\langle x\rangle$ with $\varphi_{0}$ excellent of dimension $2^{m}$, which implies that $\varphi_{0} \in G P_{m} F$, and for any $a \in F^{*}$ represented by $\varphi_{0}$ we get $\varphi \subset \varphi_{0} \otimes\langle 1, a x\rangle \in G P_{m+1} F$.

Hence, the only remaining case is $2^{m}+2 \leq \operatorname{dim} \varphi<\operatorname{dim} \psi \leq 2^{m+1}$ with $\varphi$ and $\psi$ not being Pfister neighbors. In particular, this implies $m \geq 2$. Let $\varphi_{1}$, resp. $\psi_{1}$, be the first complementary forms of $\varphi_{0}$, resp. $\psi_{0}$. We have $1 \leq \operatorname{dim} \psi_{1}<\operatorname{dim} \varphi_{1} \leq$ $2^{m}-1$. Let $\pi \in G P_{m+1} F$ be such that $\pi \cong \varphi_{0} \perp \varphi_{1}$. Let $\rho=\varphi_{1} \perp\langle-x\rangle$ and $\sigma=\psi_{1} \perp\langle-y\rangle$.

Since $\varphi$ and $\psi$ are not Pfister neighbors, it follows from Lemma 2 that $\rho$ and $\sigma$ are anisotropic and that $\varphi_{F(\rho)} \subset \pi_{F(\rho)}$, where $\pi_{F(\rho)} \in G P_{m+1} F(\rho)$ is anisotropic.

Suppose that $\varphi_{F(\psi)}$ is isotropic. Then $\varphi_{F(\psi)(\rho)}$ is isotropic. Now $F(\psi)(\rho)$ and $F(\rho)(\psi)$ are $F$-isomorphic. Hence $\varphi_{F(\rho)(\psi)}$ is isotropic and thus $\pi_{F(\rho)(\psi)}$ is also isotropic and hence hyperbolic. The Cassels-Pfister subform theorem yields that $\psi_{F(\rho)}$ is similar to a subform of $\pi_{F(\rho)}$. In particular, we have that $\psi_{F(\rho)}$ is a Pfister neighbor. By Lemma 2 we conclude that $\sigma_{F(\rho)}$ is isotropic.

Note that $2 \leq \operatorname{dim} \sigma<\operatorname{dim} \rho \leq 2^{m}$ with $m \geq 2$. Let $k \geq 1$ such that $2^{k}+1 \leq$ $\operatorname{dim} \rho \leq 2^{k+1} \leq 2^{m}$. We recall that $\sigma=\psi_{1} \perp\langle-y\rangle$ and $\rho=\varphi_{1} \perp\langle-x\rangle$ with $\varphi_{1}$, $\psi_{1}$ excellent, $\rho$ and $\sigma$ anisotropic and $\operatorname{dim} \sigma<\operatorname{dim} \rho$. We will show that $\sigma_{F(\rho)}$ is anisotropic, contradicting the isotropy of $\sigma$ over $F(\rho)$ shown above.

If $\operatorname{dim} \sigma \leq 2^{k}$, then we are in case (i) which by the above implies that $\sigma_{F(\rho)}$ is anisotropic. So suppose that $\operatorname{dim} \sigma \geq 2^{k}+1$. Then $2^{k}+2 \leq \operatorname{dim} \rho \leq 2^{k+1}$, and if we can show that $\rho$ is not a Pfister neighbor, then we are in case (ii) and the anisotropy of $\sigma_{F(\rho)}$ follows by induction because $k<m$.

So suppose $\rho=\varphi_{1} \perp\langle-x\rangle$ is a Pfister neighbor and $2^{k}+2 \leq \operatorname{dim} \rho \leq 2^{k+1}$. Since $2^{k}+1 \leq \operatorname{dim} \varphi_{1} \leq 2^{k+1}-1$, there exists a second complementary form $\varphi_{2}$ of $\varphi_{0}$ with $\operatorname{dim} \varphi_{2} \geq 1$ and $\varphi_{1} \perp \varphi_{2} \in G P_{k+1} F$. By Lemma 2, $\rho=\varphi_{1} \perp\langle-x\rangle$ being a Pfister neighbor implies that $\varphi_{2} \perp\langle x\rangle$ is isotropic. But by Lemma 1, $\varphi_{2} \perp\langle x\rangle \subset \varphi_{0} \perp\langle x\rangle=\varphi$, a contradiction to $\varphi$ being anisotropic. Hence, $\rho$ is not a Pfister neighbor.

This completes the proof of the Proposition.

Corollary. Let $F$ be formally real and $x, y \in S(F)$. Let $n>m \geq 0, \varphi=m \times\langle 1\rangle \perp$ $\langle-x\rangle$ and $\psi=n \times\langle 1\rangle \perp\langle-y\rangle$. Then $F(\psi)$ is formally real. Suppose furthermore that $\varphi$ is anisotropic. Then $\varphi_{F(\psi)}$ is anisotropic.

Proof. Since $-y<0<1$ with respect to each ordering on $F$, it follows that $\psi$ is indefinite with respect to each ordering on $F$. By [ELW, Theorem 3.5], each ordering on $F$ extends therefore to an ordering on $F(\psi)$. In particular, $F(\psi)$ is again formally real.

As already remarked, $m \times\langle 1\rangle$ and $n \times\langle 1\rangle$ are excellent forms. Thus, $\varphi$ and $\psi$ are as in the previous proposition. To establish the anisotropy of $\varphi_{F(\psi)}$ provided 
$\varphi$ is anisotropic, it therefore suffices by this proposition to verify that if $2^{k}+2 \leq$ $\operatorname{dim} \psi \leq 2^{k+1}$ for some $k \geq 1$, then $\psi$ is not a Pfister neighbor.

Suppose otherwise, i.e., suppose there exists $\pi \in P_{k+1} F$ such that $\psi$ is a Pfister neighbor of $\pi$. The subform $\left(2^{k}+1\right) \times\langle 1\rangle$ of $\psi$ is then also a Pfister neighbor of $\pi$, but it is also a Pfister neighbor of $\tau=2^{k+1} \times\langle 1\rangle \in P_{k+1} F$. Thus, $\tau \cong \pi$ and $\psi$ is in fact a subform of $\tau$ as both $\psi$ and $\tau$ represent 1 . This is impossible as $\tau$ is positive definite with respect to each ordering on $F$, whereas $\psi$ is indefinite with respect to each ordering on $F$.

Now let $F$ be a formally real field and $n \geq 1$ an integer. We define

$$
\mathcal{C}(n, F)=\{\langle\underbrace{1, \cdots, 1}_{n},-y\rangle \mid y \in S(F)\} .
$$

Proof of Theorem 1. Starting with any formally real $F_{0}$, we define the rational function field in $n-1$ variables over $F_{0}$ to be $F_{1}=F_{0}\left(t_{1}, \cdots, t_{n-1}\right)$. Put $x=$ $1+t_{1}^{2}+\cdots+t_{n-1}^{2} \in S\left(F_{1}\right)$. Then $x$ is a sum of $n$ squares in $F_{1}$ but not of $n-1$ squares (cf. [L1, Ch. 9, Cor. 2.4]), i.e. $\ell(x)=n$ over $F_{1}$. In particular, the form

$$
\varphi=\langle\underbrace{1, \cdots, 1}_{n-1},-x\rangle
$$

is anisotropic over $F_{1}$. For $i \geq 1$, we inductively define $F_{i+1}$ to be the compositum of all function fields of forms in $\mathcal{C}\left(n, F_{i}\right)$. Let $F=\bigcup_{i=0}^{\infty} F_{i}$. Then by construction and the Corollary, $F$ has the following properties:

First, $F$ is formally real (cf. also [ELW, Remark 3.6]).

Then, for each $y \in S(F)$, the form $n \times\langle 1\rangle \perp\langle-y\rangle$ is isotropic. In particular, each $y \in S(F)$ can be written as a sum of $\leq n$ squares. Hence, $p(F) \leq n$.

Finally, the form $\varphi=(n-1) \times\langle 1\rangle \perp\langle-x\rangle$ stays anisotropic over $F$. In particular, $x \in S\left(F_{1}\right) \subset S(F)$ cannot be written as a sum of $\leq n-1$ squares. Hence, $p(F) \geq n$ and therefore $p(F)=n$.

Remark. In the above proof, one has some freedom in the choice of $F_{1}$, resp. $a \in$ $F_{1}^{*}$. In fact, any formally real field extension $F_{1} / F_{0}$ and any $a \in S\left(F_{1}\right)$ will work provided $\ell(a) \geq n$.

\section{Formally Real fields with given Pythagoras numbers AND ADDITIONAL PROPERTIES}

Having constructed formally real fields with arbitrary Pythagoras number, it seems natural to ask whether our construction can be refined to yield fields with additional properties. Before continuing in this direction, let us recall the definitions of the $u$-invariant and the Hasse number $\tilde{u}$ of a field $F$ of characteristic $\neq 2$ :

$$
\begin{gathered}
u(F)=\sup \{\operatorname{dim} \varphi \mid \varphi \text { is an anisotropic torsion form } / F\}, \\
\tilde{u}(F)=\sup \{\operatorname{dim} \varphi \mid \varphi \text { is an anisotropic totally indefinite form } / F\},
\end{gathered}
$$

where torsion means that the class of $\varphi$ in the Witt ring $W F$ is torsion and totally indefinite means that $\varphi$ is indefinite with respect to each ordering on $F$ (if there are any). Recall that if $F$ is not formally real, then every form is a torsion form, and if $F$ is formally real, then by Pfister's local-global principle, torsion forms are exactly those forms $\varphi$ with signature zero with respect to each ordering on $F$ (cf. [L1, Ch. 8, Theorem 4.1], [S, Ch. 3, Theorem 6.2]). It is well known that $\tilde{u}$, resp. $u$, 
can never take the values 3,5 , or 7 (see [ELP]). For other odd values $>1$ this is still an unsolved problem. However, every even integer $n \geq 2(\geq 0$ in the formally real case) can be realized as $\tilde{u}$, resp. $u$, of a suitable field; cf. [M], [L2], [Hor].

We will assume from now on that all fields considered (unless otherwise noted) are formally real, in which case $u$, if finite, will always be even and $\tilde{u}$ will always be $\neq 1$.

Remarks and examples. (i) If $\tilde{u}(F)<\infty$ and $p(F)=1$, then $\tilde{u}(F)=0$; cf. $\quad[\operatorname{Pr}$, p. 292]. Note that the definition of $\tilde{u}$ used in $[\mathrm{Pr}]$ differs slightly from ours. It yields the same values if they are $\geq 2$, but the value 1 iff in our definition we have 0 .

(ii) For $F=\mathbb{R}((X))((Y))$, the iterated power series field in two variables over the reals, we get $p(F)=1$ and $\tilde{u}(F)=\infty$.

If $F_{0}$ is any formally real field with $p\left(F_{0}\right)=n \geq 2$, then for $F=F_{0}((X))$ we get $p(F)=n$ and $\tilde{u}(F)=\infty$.

The fact that in both cases the Pythagoras number of $F$ is as claimed follows readily from Springer's theorem for quadratic forms over a field with a discrete 2-henselian valuation (cf. [L1, Ch. 6, Sect. 1], [S, Ch. 6, Cor. 2.6]).

Over $F=\mathbb{R}((X))((Y))$, the form $n \times\langle 1\rangle \perp\langle X, Y,-X Y\rangle$ is totally indefinite and anisotropic for all $n$, and over $F=F_{0}((X))$ with $p\left(F_{0}\right)=n \geq 2$, the form $n \times\langle 1\rangle \perp X\langle 1,-a\rangle$ is totally indefinite and anisotropic for all $n$ and all $a \in S\left(F_{0}\right)$ of length $\geq 2$. (The anisotropy follows again from Springer's theorem.) Hence, $\tilde{u}(F)=\infty$ in both cases.

(iii) We have $p(\mathbb{R})=p(\mathbb{R}((X)))=1$ and $\tilde{u}(\mathbb{R})=\tilde{u}(\mathbb{R}((X)))=0$. $\mathbb{R}$ is uniquely ordered, but $\mathbb{R}((X))$ is not. On the other hand, one easily checks that if $F$ is formally real and uniquely ordered, then $p(F)=1$ implies $\tilde{u}(F)=0$.

(iv) Let $F$ be formally real with $p(F)=n \geq 2$, and let $k \geq 0$ be an integer such that $2^{k}+1 \leq n \leq 2^{k+1}$. Then $\tilde{u}(F) \geq u(F) \geq 2^{k+1}$.

In fact, $\tilde{u}(F) \geq u(F)$ always holds. Now let $a \in S(F)$ be such that $\ell(a)=$ $p(F)=n$. Then the form $(n-1) \times\langle 1\rangle \perp\langle-a\rangle$ is anisotropic, hence also the form $2^{k} \times\langle 1\rangle \perp\langle-a\rangle$, which is a Pfister neighbor of $\langle\langle-1, \cdots,-1, a\rangle\rangle \in P_{k+1} F$. This Pfister form is therefore also anisotropic, and it is furthermore torsion as $a \in S(F)$. This readily yields $u(F) \geq 2^{k+1}$. In fact, we have shown that $I_{t}^{k+1} F \neq 0$ (here, $I^{m} F$ denotes the $m$-th power of the fundamental ideal $I F$ of classes of even-dimensional forms in $W F$, and $I_{t}^{m} F$ its torsion part).

(v) The field $F=\mathbb{R}(X)$, i.e., the rational function field in one variable over the reals, has $p(F)=\tilde{u}(F)=2$. However, there do exist also uniquely ordered formally real $F$ with $p(F)=\tilde{u}(F)=2$. In fact, in [Pr, Theorem 3.1], Prestel constructs uniquely ordered fields $F$ such that $p(F)=2$ and $\tilde{u}(F)=2^{m}$ to any given integer $m \geq 1(\operatorname{resp} . \tilde{u}(F)=\infty)$.

As remarked above, all uniquely ordered fields with $p(F)=1$ have $\tilde{u}(F)=0$. Our aim will be to construct to any given integers $n \geq 2$ and $m \geq 2$ uniquely ordered fields with $p(F)=n$ and $\tilde{u}(F)=2 m$, provided these values are such that the obvious restriction given in (iv) above is satisfied, thus extending considerably Prestel's list of examples mentioned in the above remark under (v). More precisely:

Theorem 2. Let $n \geq 2, m \geq 2$ and $k \geq 0$ be integers such that $2 m \geq 2^{k+1} \geq$ $n \geq 2^{k}+1$. Then there exists a uniquely ordered field $F$ such that $p(F)=n$ and $\tilde{u}(F)=u(F)=2 m$. 
Proof. Let $F_{0}$ be any formally real field, and let

$$
F_{1}=F_{0}\left(x_{1}, \cdots, x_{n-1}, y_{1}, \cdots, y_{m-1}, z_{1}, \cdots, z_{m-1}\right)
$$

be the rational function field in $n+2 m-3$ variables over $F_{0}$. Let $a=1+x_{1}^{2}+$ $\cdots+x_{n-1}^{2}$. Then, as in the proof of Theorem $1, \ell(a)=n$ (over $F_{1}$ ), and we have the anisotropic form $\varphi$ defined by

$$
\varphi=\langle\underbrace{1, \cdots, 1}_{n-1},-a\rangle .
$$

Consider the multiquaternion algebra

$$
A=\left(1+y_{1}^{2}, z_{1}\right) \otimes \cdots \otimes\left(1+y_{m-1}^{2}, z_{m-1}\right)
$$

over $F_{1}$. Then $A$ is a division algebra over $F_{1}$ and it will stay a division algebra over $F_{1}(\sqrt{-1})$ (see, e.g., [H3, Lemma 2]). Furthermore, there exists a form $\alpha \in I^{2} F_{1}$ of dimension $2 m$ such that in $W F_{1}$ we can write

$$
\alpha \sim \lambda_{1}\left\langle\left\langle 1+y_{1}^{2}, z_{1}\right\rangle\right\rangle+\cdots+\lambda_{m-1}\left\langle\left\langle 1+y_{m-1}^{2}, z_{m-1}\right\rangle\right\rangle
$$

for suitable $\lambda_{i} \in F_{1}^{*}$, and this form $\alpha$ has the property that if $K / F_{1}$ is any field extension such that $A_{K}$ is a division algebra, then $\alpha_{K}$ is anisotropic (see, e.g., [M, Lemmas 4, 5] or [H3, Lemma 2]). In particular, $\alpha$ is anisotropic over $F_{1}$.

If $K / F_{1}$ is any formally real field extension, then, since $1+y_{i}^{2} \in S(K)$, the 2 -fold Pfister forms $\left\langle\left\langle 1+y_{i}^{2}, z_{1}\right\rangle\right\rangle$ are all torsion so that $\alpha_{K}$ is torsion as well.

Now let $P_{1}$ be any ordering on $F_{1}$ and let $M / F_{1}$ be any (formally real) field extension such that $P_{1}$ extends to an ordering $Q$ on $M$. We define

$$
\left.\mathcal{C}_{1}(M, Q)=\{\underbrace{\langle 1, \cdots, 1}_{n},-b\rangle \mid b>_{Q} 0\right\},
$$

$$
\mathcal{C}_{2}(M, Q)=\{\psi \mid \psi \text { is a totally indefinite form } / M, \operatorname{dim} \psi=2 m+1\} .
$$

Suppose now that $K$ is a (formally real) field extension of $F_{1}$ such that $P_{1}$ extends to an ordering $P$ on $K$, such that $\varphi_{K}$ is anisotropic and such that $A_{K(\sqrt{-1})}$ is a division algebra. We claim that all these properties carry over to $K(\psi)$ for any $\psi$ in $\mathcal{C}_{1}(K, P) \cup \mathcal{C}_{2}(K, P)$.

Now any such $\psi$ is by definition indefinite at $P$, hence $P$ extends to an ordering $P^{\prime}$ on $K(\psi)$ which obviously extends the ordering $P_{1}$.

If $\psi \in \mathcal{C}_{1}(K, P)$, then $\varphi$ stays anisotropic over $K(\psi)$ (the same argument as in the proof of Theorem 1 applies). If $\psi \in \mathcal{C}_{2}(K, P)$, then $\operatorname{dim} \varphi \leq 2^{k+1}<\operatorname{dim} \psi$ and the anisotropy of $\varphi_{K(\psi)}$ follows from [H1, Theorem 1].

Now if $\psi \in \mathcal{C}_{1}(K, P)$, then $\psi$ is isotropic over $K(\sqrt{-1})$ because $\langle 1,1\rangle \subset \psi$. Hence, $K(\sqrt{-1})(\psi)$ is purely transcendental over $K(\sqrt{-1})$, and so the division algebra $A_{K(\sqrt{-1})}$ will stay a division algebra over $K(\sqrt{-1})(\psi)=K(\psi)(\sqrt{-1})$. If $\psi \in$ $\mathcal{C}_{2}(K, P)$, then $A_{K(\sqrt{-1})}$ will stay a division algebra over $K(\sqrt{-1})(\psi)=K(\psi)(\sqrt{-1})$ as a consequence of Merkurjev's index reduction theorems [M, Cor. 1].

We now proceed as in the proof of Theorem 1. Starting with $F_{1}$ and any ordering $P_{1}$ on $F_{1}$, we construct for every $i \geq 1$ a field $F_{i+1}$ and an ordering $P_{i+1}$ as follows. Let $F_{i+1}$ be the compositum of all function fields of forms in $\mathcal{C}_{1}\left(F_{i}, P_{i}\right) \cup \mathcal{C}_{2}\left(F_{i}, P_{i}\right)$. By the above, $P_{i}$ extends to an ordering on $F_{i+1}$. Let $P_{i+1}$ be any ordering on $F_{i+1}$ extending $P_{i}$.

Let $F=\bigcup_{i=1}^{\infty} F_{i}$ and $P=\bigcup_{i=1}^{\infty} P_{i}$. One checks readily that $P$ defines an ordering on $F$. 
As in the proof of Theorem $1, \varphi$ stays anisotropic over $F$ and thus $p(F) \geq n$. On the other hand, all forms of type $n \times\langle 1\rangle \perp\langle-b\rangle$ with $b>_{P} 0$ are isotropic by construction, in particular also those with $b \in S(F)$. Hence $p(F) \leq n$ and thus $p(F)=n$.

By construction, all totally indefinite forms of dimension $2 m+1$ are isotropic, and since $2 m+1 \geq 5$, it follows that all totally indefinite forms of dimension $\geq 2 m+1$ are isotropic (see [ELP, Theorem A]). Hence $\tilde{u}(F) \leq 2 m$.

On the other hand, $A$ will stay a division algebra over $F(\sqrt{-1})$ and thus over $F$. Hence, the torsion form $\alpha$ of dimension $2 m$ will stay anisotropic over $F$ and thus $u(F) \geq 2 m$. All this yields $\tilde{u}(F)=u(F)=2 m$.

It remains to show that $P$ is the unique ordering on $F$. Suppose there exists an ordering $Q$ on $F, Q \neq P$. Let $b \in F^{*}$ such that $b>_{P} 0$ and $b<_{Q} 0$. Let $i \geq 1$ such that $b \in F_{i}$. Now $P$ is an ordering extending $P_{i}$, thus $b>_{P_{i}} 0$. Let $Q_{i}=Q \cap F_{i}$ and $Q_{i+1}=Q \cap F_{i+1}$. Then $Q_{i}$ is an ordering on $F_{i}$ which extends to the ordering $Q_{i+1}$ on $F_{i+1}$. In the compositum $F_{i+1}$ there appears by definition of this compositum the function field of $n \times\langle 1\rangle \perp\langle-b\rangle$. Now this form is definite at $Q_{i}$ as $b<_{Q_{i}} 0$, and hence $Q_{i}$ does not extend to an ordering on $F_{i+1}$, contradicting the fact that $Q_{i+1}$ is such an extension. Thus, $P$ is the unique ordering on $F$.

Theorem 3. Let $n$ be an integer $\geq 2$. Then there exists a uniquely ordered field $F$ with $p(F)=n$ and $\tilde{u}(F)=u(F)=\infty$.

Proof. Only slight modifications of the previous proof will be necessary. Again, we let $F_{0}$ be any formally real field and we put

$$
F_{1}=F_{0}\left(x_{1}, \cdots, x_{m-1}, y_{1}, y_{2}, \cdots, z_{1}, z_{2}, \cdots\right),
$$

but this time with an infinite number of variables $y_{i}, z_{i}$. We let $a$ be as before and we now denote the division algebra $A$ above by $A_{m-1}$, referring to the number of quaternion algebra factors. Similarly, $\alpha$ will be $\alpha_{m-1}$. We repeat the above construction, but this time only with $\mathcal{C}_{1}\left(F_{i}, P_{i}\right)$. By a similar reasoning as before, it follows that the limit $F$ will be uniquely ordered, $p(F)=n$, and that $A_{m-1}$ will be a division algebra over $F$ for all $m \geq 2$. In particular, $\left(\alpha_{m-1}\right)_{F}$ will be an anisotropic $2 m$-dimensional torsion form for all $m \geq 2$, hence $u(F)=\tilde{u}(F)=\infty$. Note that this time it is not even necessary to invoke Merkurjev's index reduction theorems.

Remark. Very little is known about the relationship between $u$ and $\tilde{u}$ in the case of a formally real field with finite $\tilde{u}$. It is well known that they will always be equal if $\tilde{u} \leq 4$. However, they need not be the same if $\tilde{u} \geq 6$, but the only known counterexamples are with $\tilde{u}=2 m+2$ and $u=2 m$, where $m$ can be any given integer $\geq 2$; cf. [Hor], [L2]. By fusing our construction in the proof of Theorem 2 with the one given in [L2] in a suitable way, we were able to construct examples of uniquely ordered $F$ with $p(F)=n, u(F)=2 m$ and $\tilde{u}(F)=2 m+2$ provided that $n \geq 4$ and there exists an integer $k \geq 2$ such that $2 m \geq 2^{k} \geq n$. We will refrain from giving the rather technical details. It would be interesting to know if such examples exist also for $p(F)=3$ or $p(F)=2$. Note that by [EP, Prop. 2.7], $p(F)=2$ together with $\tilde{u}(F)$ finite implies $u(F) \leq \tilde{u}(F) \leq u(F)+2$. One can even show (we leave this to the reader) that in this situation, $u(F)=\tilde{u}(F)$ provided $u(F) \leq 6$ or $\tilde{u}(F) \leq 8$. 


\section{REFERENCES}

[A] E. Artin, Über die Zerlegung definiter Funktionen in Quadrate, Abh. Math. Semin. Hamburg. Univ. 5 (1927) 100-115.

[CEP] J.W.S. Cassels, W.J. Ellison, and A. Pfister, On sums of squares and on elliptic curves over function fields, J. Number Theory 3 (1971) 125-149. MR 45:1863

[EL] R. Elman, T.Y. Lam, Pfister forms and K-theory of fields, J. Algebra 23 (1972) 181-213. MR 46:1882

[ELP] R. Elman, T.Y. Lam, and A. Prestel, On some Hasse principles over formally real fields. Math. Z. 134 (1973) 291-301. MR 48:8384

[ELW] R. Elman, T.Y. Lam, and A.R. Wadsworth, Orderings under field extensions, J. Reine Angew. Math. 306 (1979) 7-27. MR 80e:12029

[EP] R. Elman and A. Prestel, Reduced stability of the Witt ring of a field and its Pythagorean closure, Amer. J. Math. 106 (1983) 1237-1260. MR 86f:11030

[H1] D.W. Hoffmann, Isotropy of quadratic forms over the function field of a quadric, Math. Z. 220 (1995) 461-476. MR 96k:11041

[H2] , Twisted Pfister forms, Doc. Math. J. DMV 1 (1996) 67-102. MR 97d:11065

[H3] $\quad$ On Elman and Lam's filtration of the u-invariant, J. Reine Angew. Math. 495 (1998), 175-186. MR 99d:11037

[Hor] E.A.M. Hornix, Formally real fields with prescribed invariants in the theory of quadratic forms, Indag. Math. 2 (1991) 65-78. MR 92f:11055

[I] O.T. Izhboldin, On the isotropy of quadratic forms over the function field of a quadric, Algebra i Analiz. 10 (1998), 32-57. (Russian). English transl. to appear in St. Petersburg Math. J. 10 (1999). CMP 98:12

[K] M. Knebusch, Generic splitting of quadratic forms. II, Proc. London Math. Soc. 34 (1977) 1-31. MR 55:379

[L1] T.Y. Lam, The Algebraic Theory of Quadratic Forms, Reading, Massachusetts: Benjamin 1973 (revised printing 1980). MR 53:277

[L2] _ Some consequences of Merkurjev's work on function fields, Preprint 1989.

[M] A.S. Merkurjev, Simple algebras and quadratic forms, Izv. Akad. Nauk. SSSR 55 (1991) 218-224. (English translation: Math. USSR Izvestiya 38 (1992) 215-221.) MR 93b:16025

[Pe] M. Peters, Summe von Quadraten in Zahlringen, J. Reine Angew. Math. 268/269 (1974) 318-323. MR 50:4551

[Pf] A. Pfister, Quadratic forms with applications to algebraic geometry and topology, London Math. Soc. Lect. Notes 217, Cambridge University Press 1995. MR 97c:11046

[Pr] A. Prestel, Remarks on the Pythagoras and Hasse number of real fields, J. Reine Angew. Math. 303/304 (1978) 284-294. MR 80d:12020

[Sc] R. Scharlau, On the Pythagoras number of orders in totally real number fields, J. Reine Angew. Math. 316 (1980) 208-210. MR 81g:10036

[S] W. Scharlau, Quadratic and Hermitian Forms, Grundlehren 270, Berlin, Heidelberg, New York, Tokyo: Springer 1985. MR 86k:11022

Equipe de Mathématiques de Besançon, UMR 6623 du CNRS, Université de FrancheComté, 16, Route de Gray, F-25030 Besançon Cedex, France

E-mail address: detlev@math.univ-fcomte.fr 